\title{
Targeting Human Telomerase Reverse Transcriptase by a Simple siRNA Expression Cassette in HepG2 Cells
}

\author{
Hui Xu ${ }^{1}$; Xia Gong ${ }^{1}$; Hui Hui Zhang ${ }^{1}$; Qin Zhang ${ }^{1}$; Dandan Zhao ${ }^{1}$; Jian Xiong Peng ${ }^{1, *}$ \\ ${ }^{1}$ Department of Medical Laboratory, Xiangya Medial School, Central South University (CSU), Changsha, China \\ ${ }^{*}$ Corresponding Author: Jian Xiong Peng, Department of Medical Laboratory, Xiangya Medial School, Central South University (CSU), Changsha, China. Tel: +88-60731-82650279, \\ E-mail: pengjianxiong66@163.com
}

Received: October 8, 2014; Revised: January 3, 2015; Accepted: February 21, 2015

\begin{abstract}
Background: Human telomerase reverse transcriptase (hTERT) has become an ideal target for development of anticancer therapy. Small interfering RNAs (siRNAs) are very powerful reagents for gene silencing and show promise for cancer gene therapy. However, only a small number of siRNAs have been demonstrated to be effective. For gene therapy targeting hTERT, it is essential to develop a robust system to fully explore the power of siRNAs.

Objectives: We explored a siRNA expression cassette (SEC) to screen highly effective RNAi-targeted sequences for gene therapy of hepatocellular carcinoma (HCC).

Materials and Methods: An SEC was developed by flanking H1 and U6 promoters in opposite directions at the siRNA-encoding sequence. Eight SECs specific to hTERT were designed by overlap extension polymerase chain reaction (PCR) and transfected into HepG2 cells with calcium phosphate. The telomerase activity was determined by telomeric repeat amplification protocol (TRAP) silver staining and TRAP real-time PCR analysis. The mRNA and protein expression levels of hTERT were determined by reverse transcription (RT)-PCR and western blot, respectively. Cell viability was determined by the 3-(4,5-dimethylthiazol-2-yl)-2, 5-diphenyltetrazolium bromide (MTT) assay and cell apoptosis was measured by the annexin-V/propidium iodide(PI) assay coupled with flow cytometry.

Results: Eight hTERT-specific SECs (SEC-1-8) were successfully constructed. In comparison to that of the negative control SEC, the hTERTspecific SECs, especially, SEC-4, SEC-5, SEC-7 and SEC-8 significantly reduced the activity of hTERT in HepG2 cells at 48 hours after transfection. Moreover, the mRNA and protein expression levels of hTERT as well as the cell viability were significantly reduced by SECs. Knockdown of hTERT by SECs in HepG2 cells led to cell apoptosis.

Conclusions: Our developed simple SEC was a powerful strategy for screening highly effective RNAi-targeted sequences and showed promise for gene therapy of HCC.

Keywords: hTERT; siRNA; Telomerase; Hepatocellular Carcinoma; Gene Therapy
\end{abstract}

\section{Background}

Human telomerase reverse transcriptase (hTERT) is the rate-limiting enzyme for the maintenance of telomeres of chromosomes. The activity of hTERT is repressed in normal somatic tissues, but becomes reactivated in 8590\% of human malignancies, including hepatocellular carcinoma (HCC) (1). It is widely accepted that deregulation of hTERT plays key roles during multistep processes of tumorigenesis and anticancer drug resistance. Accordingly, hTERT has become an important target for development of anticancer therapies, with numerous agents in both preclinical and clinical trials. Currently, there are a number of compounds that target hTERT, including papaverine (2), $\beta$-carboline alkaloid harmine (3), crocin (4) and the dominant-negative mutant of hTERT (DN-hTERT) (5).

RNA interference (RNAi) with small interfering RNA (siRNA) has been proven a powerful tool for gene knockdown through directed post-transcriptional gene silencing. Imani Fooladi et al. found potential use of siRNA for prophylaxis and treatment of Pseudomonas aeruginosainduced hematogenous pulmonary infection in human (6). At present, siRNA is achieved by either chemical synthesis or siRNA-expressing vectors (7). However, chemically synthesized siRNA is expensive, thus preventing its large-scale application in preclinical and clinical trials; while vector-delivered siRNA is tedious for screening highly effective RNAi-targeted sequences and has longterm safety risks for clinical applications.

To overcome limitations of the cost of chemically synthesized siRNA and time-consuming synthesis of vectordelivered siRNA, the concept of siRNA expression cassettes (SECs) has been explored.SECs are siRNA expression templates obtained by polymerase chain reaction (PCR) and can be delivered into cells directly without cloning into expression vectors. SECs were first constructed by Castanotto et al. through four rounds of nested PCR (8). Similarly, Gou et al. used a two-step PCR procedure to generate a chimeric DNA template containing U6 promoter 
and a short hairpin DNA (9). However, the disadvantage of a short hairpin RNA (shRNA) expression cassette is that two complimentary long oligoribonucleotides, which include sense, loop and antisense sequences, have to be chemically synthesized. Chemical synthesis of these long oligoribonucleotides is very expensive and error-prone. Tran et al. co-expressed the sense and antisense RNAs using dual U6 promoters (10); nevertheless, application of the same U6 promoters might lead to unwanted homologous recombination, hence limiting its efficiency.

\section{Objectives}

Here, we reported the construction of SECs that contain two distinct DNA polymerase III promoters, human $\mathrm{H} 1$ and U6 promoters, to drive the expression of the sense and antisense strands of the siRNA, respectively. Our results demonstrated that these SECs specific to hTERT efficiently inhibited both the mRNA and protein expression of hTERT in HepG2 cells. Moreover, they reduced the cell viability and induced cell apoptosis.

\section{Materials and Methods}

\subsection{Construction of SECs With Two Convergent Pol III Promoters}

The plasmids pSUPER retro Neo + GFP with the H1 promoter and pRNAT-U6.1/Neo with the U6 promoter were from The Cancer Institute of Central South University
(Changsha, China). The overlap extension PCR was designed to produce an SEC from the above two plasmids (Figure 1). These PCRs needed four primers. Two universal U6 forward and $\mathrm{H} 1$ forward primers were used in all PCR amplifications. The other two specific primers (U6 reverse primers and $\mathrm{H} 1$ reverse primers) were designed to contain the following sequences; a 19-nt siRNA targeting sequence, termination signal sequences of the other promoter and a promoter-specific sequence, which could anneal to the end of the template plasmid during PCR amplification.

The last five nucleotides of each promoter were substituted by "-CTTTT-" and "-TTTTT-", which served as the termination signals for the $\mathrm{H} 1$ and U6 promoters, respectively. The specific 19-nt siRNA targeting sequence was designed from the hTERT mRNA (NM-198253) by the BLOCK-iT ${ }^{\mathrm{TM}}$ RNAi Designer. The specific targeting site and its corresponding primer sequences are listed in Table 1.

PCR 1 and PCR 2 were performed with Taq Master Mix (OMEGA, USA) under the following conditions; 3 minutes at $94^{\circ} \mathrm{C}, 30$ seconds at $95^{\circ} \mathrm{C}, 30$ seconds at $55^{\circ} \mathrm{C}$ and $1 \mathrm{~min}$ ute at $72^{\circ} \mathrm{C}$ for 32 cycles followed by incubation at $72^{\circ} \mathrm{C}$ for 5 minutes. PCR 3 was performed with ExTaq DNA polymerase (TaKaRa, Japan) under the following conditions; 3 minutes at $94^{\circ} \mathrm{C}, 30$ seconds at $95^{\circ} \mathrm{C}, 30$ seconds at $50^{\circ} \mathrm{C}$ and $1 \mathrm{~min}$ utes at $72^{\circ} \mathrm{C}$ for five cycles (without ExTaq DNA polymerase) followed by 30 seconds at $95^{\circ} \mathrm{C}, 30$ seconds at $66^{\circ} \mathrm{C}$ and 1 minutes at $72^{\circ} \mathrm{C}$ for 28 cycles and incubation at $72^{\circ} \mathrm{C}$ for 5 minutes (with ExTaq DNA polymerase). The PCR products were purified with a PCR purification kit (Thermo, USA) and confirmed by sequencing (Shaenggong, China).

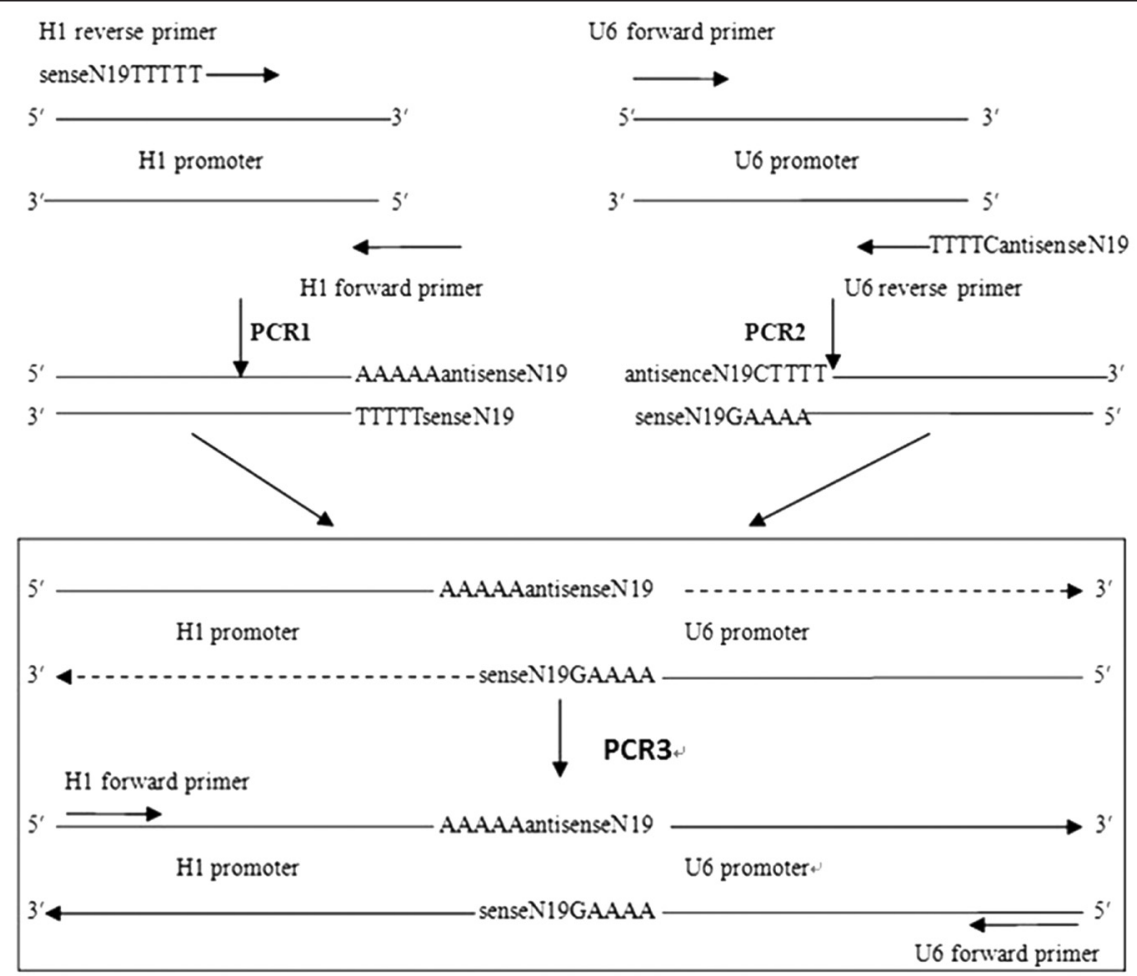

Figure 1. Schematic Representation of the Overlap Extension PCR Amplification With DNA Pol III Promoters H1 and U6 
Xu Het al.

\begin{tabular}{|c|c|c|}
\hline Name & Sequence & Targeting Location \\
\hline H1F & 5'-GAA TTC GAA CGC TGA CGT C-3' & H1 forward \\
\hline U6F & 5'-GAG AAG CAT GAA TTC CCC AGT-3' & U6 forward \\
\hline H1R-1 & 5'-AAG ATG GCC TTC TCA CAG ATT TTT GTG GTC TCA TAC AGA AC-3' & 1778-1796 \\
\hline U6R-1 & 5'-TCT GTG AGA AGG CCA TCT TCT TTT CGC GTC CTT TCC ACA AGA TA-3' & 1778-1796 \\
\hline H1R-2 & 5’-GGA GCA AGT TGC AAA GCA TTT TTT GTG GTC TCA TAC AGA AC-3' & 1800-1818 \\
\hline U6R-2 & 5’-ATG CTT TGC AAC TTG CTC CCT TTT CGC GTC CTT TCC ACA AGA TA-3' & $1800-1818$ \\
\hline H1R-3 & 5'-GGG CTG CGG CCG ATT GTG ATT TTT GTG GTC TCA TAC AGA AC-3' & 1943-1961 \\
\hline U6R-3 & 5'-TCA CAA TCG GCC GCA GCC CCT TTT CGC GTC CTT TCC ACA AGA TA-3' & 1943-1961 \\
\hline H1R-4 & 5'-GCG TTT GGT GGA TGA TTT CTT TTT GTG GTC TCA TAC AGA AC-3' & $2650-2668$ \\
\hline U6R-4 & 5'-GAA ATC ATC CAC CAA ACG CCT TTT CGC GTC CTT TCC ACA AGA TA-3' & $2650-2668$ \\
\hline H1R-5 & 5'-GGA AGA CAG TGG TGA ACT TTT TTT GTG GTC TCA TAC AGA AC-3' & $2760-2778$ \\
\hline U6R-5 & 5'-AAG TTC ACC ACT GTC TTC CCT TTT CGC GTC CTT TCC ACA AGA TA-3 & $2760-2778$ \\
\hline H1R-6 & 5'-GCC TGT TTC TGG ATT TGC ATT TTT GTG GTC TCA TAC AGA AC-3' & 3009-3027 \\
\hline U6R-6 & 5'-TGC AAA TCC AGA AAC AGG CCT TTT CGC GTC CTT TCC ACA AGA TA-3' & 3009-3027 \\
\hline H1R-7 & 5'-GGG AAT TTG GAG TGA CCA ATT TTT GTG GTC TCA TAC AGA AC-3' & 3878-3896 \\
\hline U6R-7 & 5’-TTG GTC ACT CCAAAT TCC CCT TTT CGC GTC CTT TCC ACA AGA TA-3’ & 3878-3896 \\
\hline H1R-8 & 5'-GAG GTG CTG TGG GAG TAA ATT TTT GTG GTC TCA TAC AGA AC-3' & 3964-3982 \\
\hline U6R-8 & 5’-TTT ACT CCC ACA GCA CCT CCT TTT CGC GTC CTT TCC ACA AGA TA-3’' & 3964-3982 \\
\hline H1R-NC & 5'-GTG TGA TGT GTC GGT ATT CTT TTT GTG GTC TCA TAC AGA AC-3' & Negative control \\
\hline U6R-NC & 5'-GAA TAC CGA CAC ATC ACA CCT TTT CGC GTC CTT TCC ACA AGA TA-3' & Negative control \\
\hline
\end{tabular}

$\mathrm{a}$ The TTTTT and CTTTT flanking the underlined sequences served as the U6 promoter and H1 promoter termination signals, respectively.

\subsection{Cell Culture and Transfection}

HepG2 cells were cultured in Dulbecco's modified Eagle medium (Hyclone, USA) supplemented with $10 \%$ fetal bovine serum at $37^{\circ} \mathrm{C}$ in a $\mathrm{CO}_{2}$ incubator. The cells were transfected with SECs using calcium phosphate reagents (11). Each experiment was repeated three times.

\subsection{Telomeric Repeat Amplification Protocol (TRAP) With Silver Staining}

TRAP is a standard method for detection of telomerase activity. Unlike western blotting, which detects the relative protein expression levels, TRAP directly detects the activity of proteins. At 48 hours after transfection, telomerase activity was determined by TRAP with two primers (TS, 5'-AAT CCG TCG AGC AGA GTT-3' and ACX, 5'-GCGCGG[CTTACC]3CTAACC-3') (12). The cycling program was $25^{\circ} \mathrm{C}$ for 30 minutes, $94^{\circ} \mathrm{C}$ for 3 minutes (without Taq DNA polymerase and ACX) followed by $95^{\circ} \mathrm{C}$ for 30 seconds, $55^{\circ} \mathrm{C}$ for 30 seconds and $72^{\circ} \mathrm{C}$ for 1 minute (33 cycles, with Taq DNA polymerase and ACX). PCR products were analyzed by polyacrylamide gel electrophoresis (PAGE) with silver staining. The PAGE gel was grayscale scanned by Quantity One software (Bio-Rad, USA) on each lane.

\subsection{TRAP With Real-Time PCR}

SYBR Green (Thermo, USA) was used for absolute quantification of telomerase activity with two primers (TS, 5'-AAT CCG TCG AGC AGA GTT-3' and ACX, 5'-GCGCGG[CTTACC]3CTAACC-3'). The standard TSR 8 (5'-AA TCCGTCGAGCAGAGTTAG[GGTTAG]7-3') (13) was diluted to eight different concentrations (amol $/ \mu \mathrm{L}): 5,1,0.2,0.04$, $0.008,0.0016,0.00032$ and 0.000064 . Real-time PCR was performed under the following conditions; 30 minutes at $25^{\circ} \mathrm{C}, 10$ minutes at $95^{\circ} \mathrm{C}, 15$ seconds at $95^{\circ} \mathrm{C}$ and 30 seconds at $60^{\circ} \mathrm{C}$ for 40 cycles.

\subsection{Reverse Transcription (RT)-PCR}

TRIzol reagent (Thermo, USA) was used for total RNA extraction. An RT-PCR kit (Thermo, USA) was used for the RT reaction. Quantitative analysis was performed using $\beta$-actin as an internal reference. The primers for hTERT were as follows; forward, 5'-AAGTTCCTGCACTGGCTGATG-3'; reverse, 5'-GCTTTGCAACTTGCTCCAGAC-3'. For $\beta$-actin: forward, 5'-TGACGTGGACATCCGCAAAG-3'; reverse, 5'-CTGGAAGGTGGACAGCGAGG-3'. The cycling program was $95^{\circ} \mathrm{C}$ for 2 minutes, $95^{\circ} \mathrm{C}$ for 20 seconds, $62^{\circ} \mathrm{C}$ for 20 seconds and $72^{\circ} \mathrm{C}$ for 20 seconds (40 cycles). The relative level of RNA was computed using the $2^{-\Delta \Delta \mathrm{Ct}}$ analysis method.

\subsection{Western Blot}

Total proteins from cells were extracted on ice with cell lysis buffer (Cell Signaling Biotechnology, USA). Equal amounts of proteins $(20 \mu \mathrm{g})$ were separated by 
Xu Het al.

$8 \%$ sodium dodecyl sulfate (SDS)-PAGE and transferred onto polyvinylidene difluoride (PVDF) membranes (Millipore, USA). The membranes were blocked with 5\% fat-free milk in phosphate-buffered saline (PBS) at room temperature for one hour and probed with primary antibodies (hTERT, 1:1000, Abcam; $\beta$-actin, 1:2000, Proteintech Group, USA) overnight at $4^{\circ} \mathrm{C}$. Then, the membrane was incubated with horseradish peroxidase-conjugated secondary antibody (Abcam) at room temperature for 45 minutes. Reactive bands were detected using enhanced chemiluminescence reagents (Amersham Pharmacia Biotech, USA).

\subsection{3-(4, 5-Dimethylthiazol-2-yl)-2, 5-Diphenyltet- razolium Bromide (MTT) Assay}

After transfection, cells were cultured in 96-well plates for 1-5 days. The cell viability was analyzed by the MTT assay. At the indicated time points, $20 \mu \mathrm{L}$ of MTT solution $(5 \mathrm{mg} / \mathrm{mL}$ ) was added to each well and incubated at $37^{\circ} \mathrm{C}$ for four hours. Then, the medium was replaced with $200 \mu \mathrm{L}$ of dimethyl sulfoxide. The absorbance of each well was measured in an enzyme immunoassay analyzer at $490 \mathrm{~nm}$.

\subsection{Annexin-V/Propidium Iodide (PI) Assay}

At 48 hours after transfection, cells were collected and subjected to annexin V/PI double staining according to the manufacturer's instructions (Keygen Biotech, China). The samples were then analyzed using a FACS scanner (Becton Dickinson) and the FlowJo software (Tree Star, Ashland, OR, USA).

\subsection{Statistical Analysis}

All data were expressed as means \pm standard deviation and statistical analysis was performed using analysis of variance. All P values were based on two-sided hypothesis testing and $\mathrm{P}<0.05$ was considered statistically significant.

\section{Results}

\subsection{Construction and Validation of SECs With Two Convergent Pol III Promoters}

The SECs for each of the targeting sites were successfully established as confirmed by PCR analysis (Figure 2 ). The single $\mathrm{H} 1$ and U6 promoters were $245 \mathrm{bp}$ and $366 \mathrm{bp}$, respectively. The SECs were $592 \mathrm{bp}$ confirmed by DNA sequencing.

\subsection{Effect of SECS on Telomerase Activity as Deter- mined by TRAP Silver Staining}

Eight SECs specific to the target sites 1778-1796, 18001818, 1943-1961, 2650-2668, 2760-2778, 3009-3027, 3878-
3896 and 3964-3982 of hTERT were designated as SEC-1, SEC-2, SEC-3, SEC-4, SEC-5, SEC-6, SEC-7 and SEC-8, respectively (Figure $3 \mathrm{~A}$ ). These hTERT-specific SECs were produced by the above two convergent Pol III promoters PCR and transfected into HepG2 cells. Forty-eight hours after transfection, telomerase activity was determined by TRAP silver staining analysis. Compared with that of mock and negative SEC, all eight target site-specific SECs significantly decreased the telomerase activity to different degrees (Figure $3 \mathrm{~B}$ ). RNAi inhibition rates were $55.00 \pm 1.23 \%, 42.38 \pm 5.07 \%, 47.60 \pm 2.36 \%$, $78.50 \pm$ $2.48 \%, 70.95 \pm 4.18 \%, 60.35 \pm 1.67 \%, 81.80 \pm 5.82 \%$ and 79.51 $\pm 5.73 \%$, respectively, all of which were significantly different from the negative control group $(3.56 \pm 0.70 \%)(\mathrm{P}$ $<0.05$ ) (Figure 3 C). SEC-4, SEC-5, SEC-7 and SEC-8 demonstrated the strongest inhibitory effects on telomerase activity (Figure $3 \mathrm{C}$ ). These results showed that our constructed hTERT-specific SECs efficiently decreased the telomerase activity in HepG2 cells.

\subsection{Accurate Determination of Telomerase Activ- ity by TRAP Using Real-Time PCR}

To further validate our observation that hTERT-specific SECs downregulated the telomerase activity in HepG2 cells, we applied TRAP by real-time PCR to accurately determine the telomerase activity following SEC transfection. In TRAP real-time PCR analysis, telomerase RNA components as templates by themselves were reversely transcribed into the telomerase extension products, which were further determined by real-time PCR. Thus, this method directly detected the activity of proteins. After 48 hours of transfection of SEC-2, SEC-4, SEC-7 and SEC-8, the telomerase activity in HepG2 cells was substantially decreased. The amounts of telomerase extension products (copies) after SEC-2, SEC-4, SEC-7 and SEC-8 transfection were $11.69 \pm 1.16,3.70 \pm 0.29,2.17$ \pm 0.17 and $1.730 \pm 0.11$, respectively, which were significantly different from the negative control group (40.94 $\pm 2.37)(\mathrm{P}<0.05)$ (Figure 4$)$.

Figure 2. Identification of SECs by Overlap Extension PCR

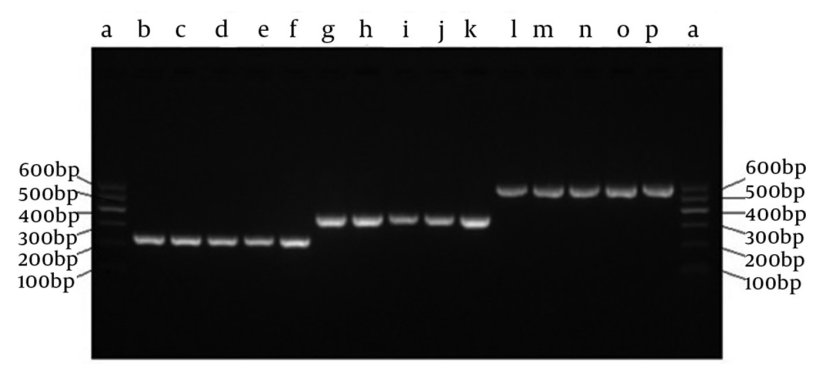

a, DNA Marker I Ladder; b-f, the single H1 promoter by PCR 1 (245 bp); g-k the single U6 promoter by PCR 2 (366 bp); l-p, the SECs by PCR 3 (592 bp). 
Xu Het al.

Figure 3. hTERT-Specific SECs Efficiently Decreased the Telomerase Activity in HepG2 Cells
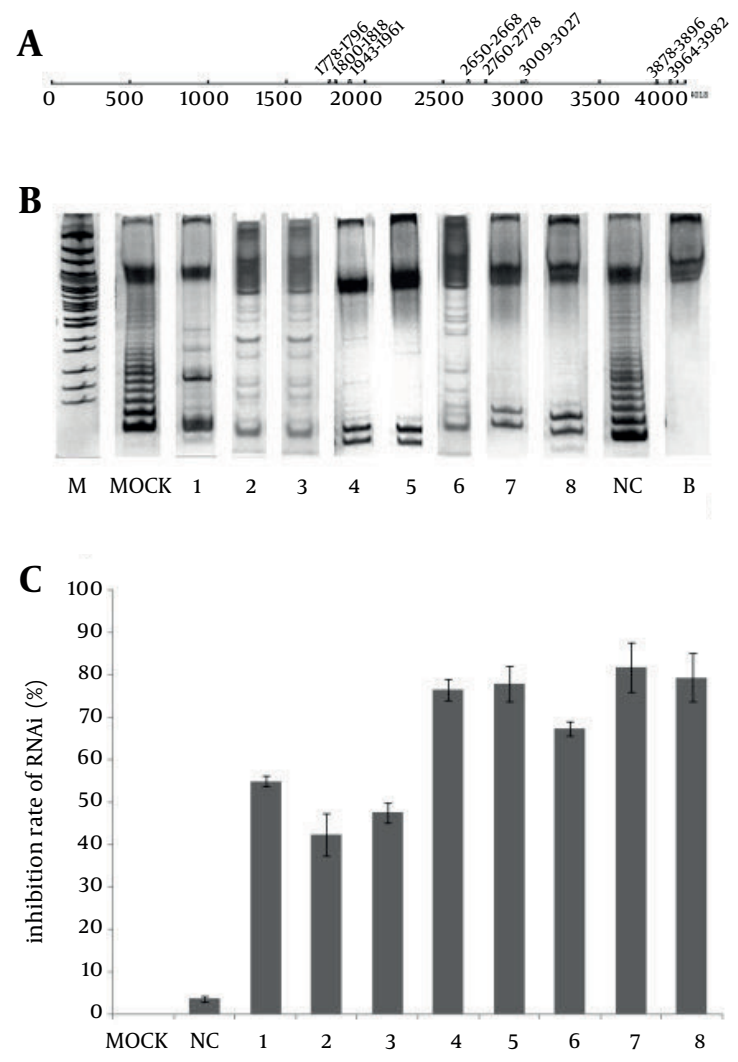

A) Target sites specific to hTERT B) HepG2 cells were transfected with the indicated SECs. Forty-eight hours later, the telomerase activity was determined by TRAP silver staining analysis. C) Inhibition rate of RNAi, Inhibition rate of $\mathrm{RNAi}(\%)=$ the relative telomerase activity of the negative control group-the relative telomerase activity of the experimental group; relative telomerase activity $=$ (scanning gray value of the experimental group/scanning gray value of the negative control group) $\times 100 \% .1-8$ : SEC1-8; M, pBR322 DNA/Mspl Marker. Mock, calcium phosphate reagent only; $\mathrm{NC}$, negative control SEC; B, Blank control.

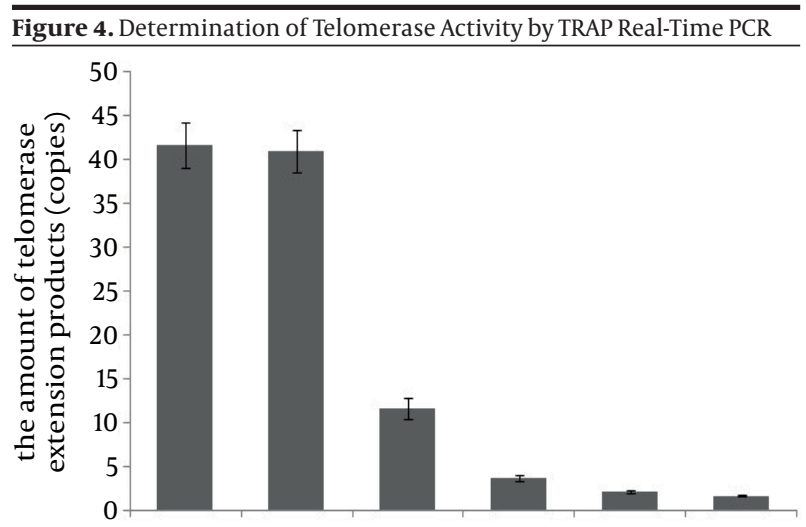

HepG2 cells were transfected with the indicated SECs. 48 hours later, the telomerase activity was determined by TRAP real-time PCR analysis. Mock, calcium phosphate reagent only; NC, negative control; 1-4, SEC-2, SEC-4, SEC-7, and SEC-8, respectively.

\subsection{SECs Reduced Both the mRNA and Protein Lev- els of hTERT}

To check whether the observed decrease of telomerase activity following SEC transfection resulted from siRNAmediated downregulation of hTERT mRNA and protein expression, we conducted RT-PCR and western blot analyses. Both the mRNA and protein expression levels of hTERT in HepG2 cells were significantly decreased 48 hours after transfection with SEC-1, SEC-3 or SEC-4. The relative expression levels of hTERT mRNA were $0.68 \pm 0.03$, $0.69 \pm 0.04$ and $0.54 \pm 0.07$ by SEC-1, SEC-3 and SEC-4, respectively, which were significantly less than by the negative control SEC $(1.10 \pm 0.11)(\mathrm{P}<0.05)$ (Figure 5).

Figure 5. hTERT-Specific SECs Decreased Both the mRNA and Protein Expression Levels of hTERT in HepG2 Cells

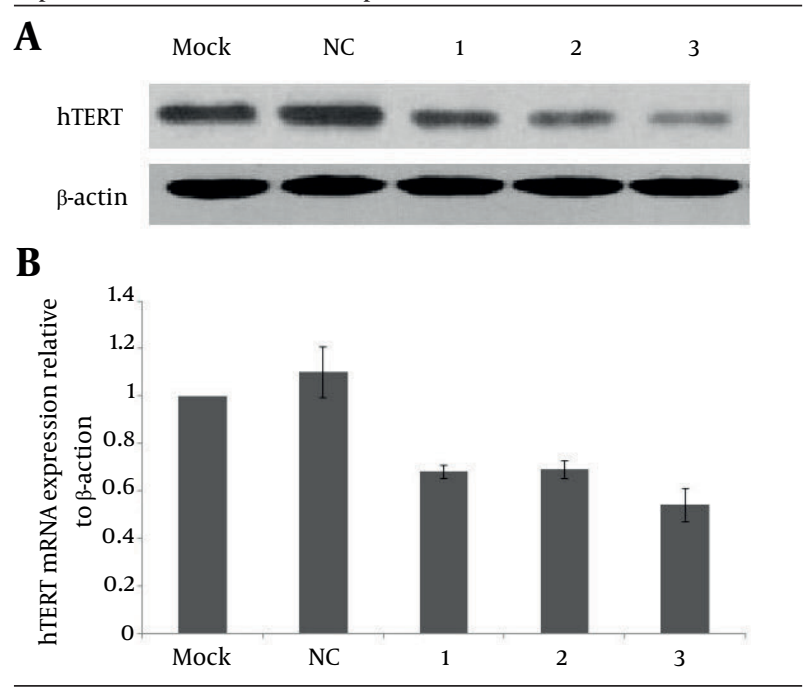

HepG2 cells were transfected with negative control, SEC-1, SEC-3 and SEC-4. A) The protein expression of hTERT was determined by western blot 48 hours later, using $\beta$-actin as the loading control B) The relative mRNA levels of hTERT were determined by real-time PCR, with $\beta$-actin as the internal control. Mock, calcium phosphate reagent only; NC, negative control; 1-3, SEC-1, SEC-3 and SEC-4, respectively.

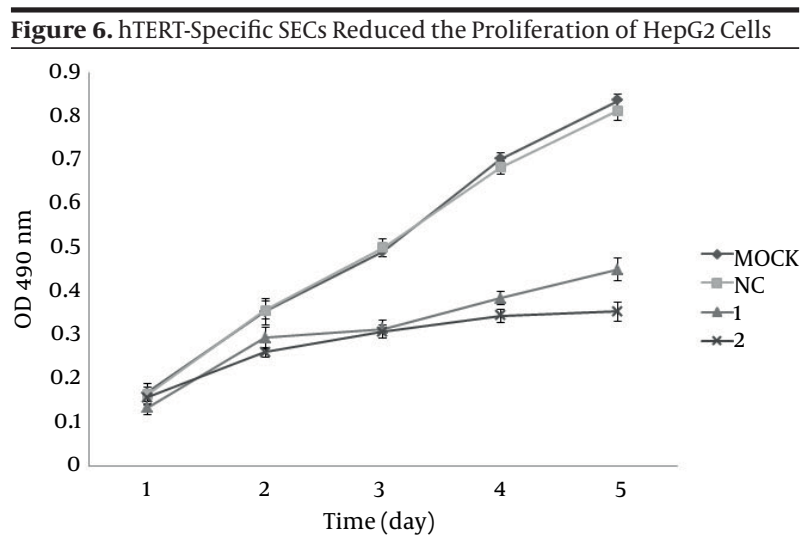

HepG2 cells were transfected with the indicated SECs or controls for 1-5 days. Cell viability was determined by the MTT assay. Mock, calcium phosphate reagent only; NC, negative control; 1 , SEC-4; 2, SEC-7. 
A
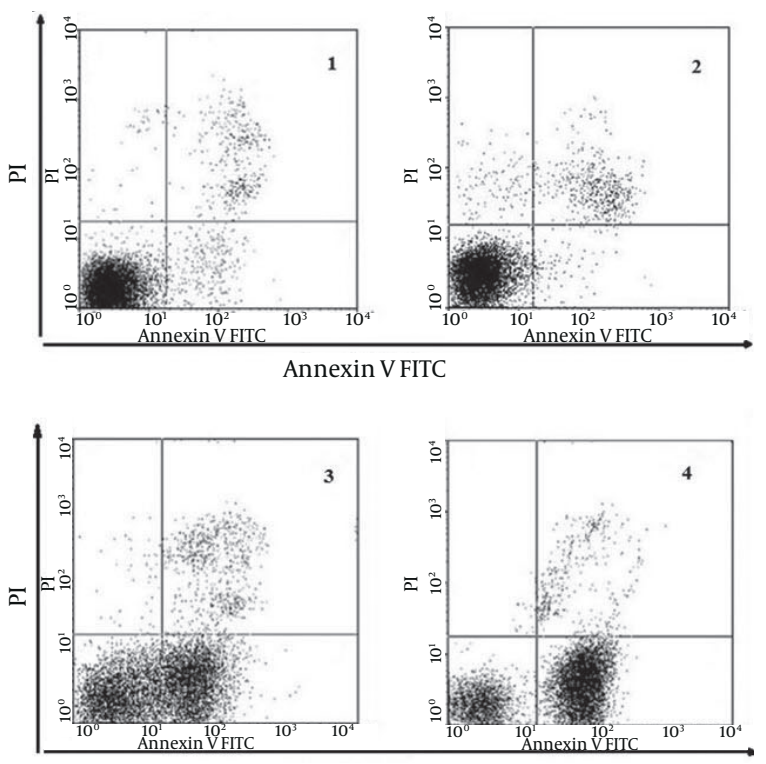

Annexin V FITC
B

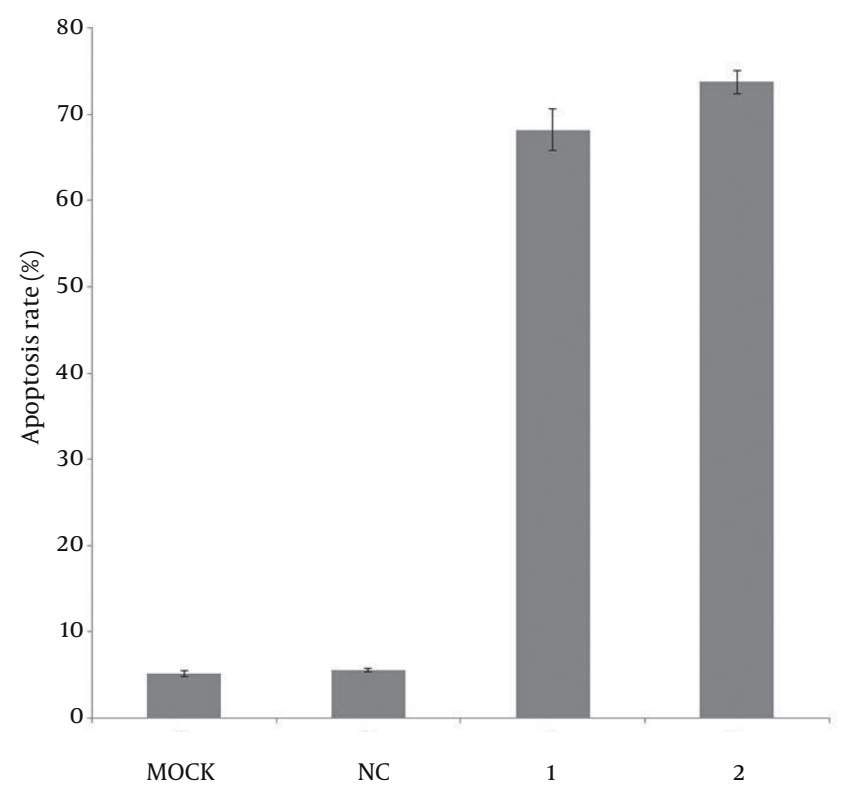

HepG2 cells were transfected with the indicated SECs or controls for 48 hours. Cell apoptosis was assessed by annexin V/PI staining coupled with flow cytometry. A) Representative histograms. 1, calcium phosphate reagent only; 2, negative control; 1, SEC-4; 2, SEC-7. B) Quantification of A. Mock, calcium phosphate reagent only; NC, negative control;1, SEC-4; 2 , SEC-7.

\subsection{SECs Targeting hTERT Inhibited the Prolifera- tion of HepG2 Cells}

To assess the effect of SECs on cell proliferation, we treated HepG2 cells with SEC-4 and SEC-7 and examined cell proliferation by the MTT assay. As shown in Figure 6, from the third day, the proliferation rates in the SEC-4 and SEC-7 groups were significantly lower than the control group $(\mathrm{P}<0.05)$.

\subsection{SECs Targeting hTERT Induced Apoptosis of HepG2 Cells}

To further investigate the effect of SECs on cell apoptosis, we then applied annexin V/PI double staining of HepG2 cells after treatment with SECs. Annexin V vs. PI plots from the gated cells showed the populations corresponding to viable (Annexin V-/PI-), necrotic (Annexin V-/PI+), early (Annexin V+/PI-) and late (Annexin V+/PI+) apoptotic cells (Figure $7 \mathrm{~A}$ ). The apoptosis rates (early apoptosis and late apoptosis) in the SEC-4 and SEC-7 groups were $68.19 \pm 2.42 \%$ and $73.73 \pm 1.34 \%$, respectively, which were significantly higher than that in the negative control group $(5.50 \pm 0.20 \%)(\mathrm{P}<0.05)$ (Figure $7 \mathrm{~B})$.

\section{Discussion}

In this study, we developed a novel SEC containing human $\mathrm{H} 1$ and U6 DNA polymerase III promoters to drive the expression of the sense and antisense strands of a
siRNA, respectively. Our results demonstrated that this type of SEC specific to hTERT efficiently inhibited both the mRNA and protein levels of hTERT in HepG2 cells. Moreover, SECs targeting hTERT inhibited cell proliferation and induced apoptosis of HepG2 cells. Our study suggested that SECs are a powerful strategy for screening highly effective RNAi-targeted sequences and showed promise for gene therapy of HCC.

El-Saeed et al. found that detection of hTERT-mRNA was superior to $\alpha$-fetoprotein in the diagnosis of chronic liver disease and could be used as a marker for early diagnosis of HCC (14). Moreover, regarding the critical roles of hTERT in tumorigenesis and anticancer drug resistance as well as powerful gene knockdown through directed posttranscriptional gene silencing, targeting hTERT by RNAi has become a promising novel strategy for the development of anticancer treatments. Zhang et al. transfected chemically synthesized siRNA directly into SiHa cells, but the efficacy of RNAi targeting hTERT was limited (15). To achieve stable and long-term RNAi, Cheng et al. (16) and Fang et al. (17) improved the RNAi efficiency using a plasmid or adenoviral vector, but construction of plasmid was tedious and exogenous viral DNA of the adenoviral vector has long-term safety risks for clinical applications. Therefore, it is essential to develop a fully robust system to optimize the efficacy of designed siRNAs.

We developed a novel and simple strategy to produce siRNA by SECs with two opposing polymerase III promot- 
ers. Once transfected into cells, gene-specific sequences could be transcribed by H1/U6 promoters to generate RNA duplexes, which would silence the corresponding gene expression. It has been reported that two different convergent promoters could increase the transcription efficiency (10); thus, we accordingly selected two different RNA Pol III promoters. Although construction of dual promoter vectors for RNAi has been reported $(18,19)$, our method of producing SECs was affordable and simple. Moreover, compared with the LineSilence ${ }^{\mathrm{TM}}$ RNAi Kit (Allele, USA), our design is more cost-effective. In our system, the two forward primers are identical for all PCRs and only the two reverse primers containing the targeting sequence need to be synthesized. Therefore, it is timesaving to screen the effective RNAi sites and safe for longterm clinical applications.

To validate our strategy, we constructed eight SECs specific to hTERT and determined their efficiency in HepG2 cells. Compared with mock and negative SECs, all eight target site-specific SECs significantly decreased the telomerase activity to different degree. Moreover to avoid a "single cell type phenomenon", we transfected SEC-4 and SEC-7 targeting hTERT in Bel-7404 and HeLa cells. In Bel-7404 cells, the RNAi inhibition rates of SEC-4 and SEC-7 were $67.49 \pm 2.06 \%$ and $77.95 \pm 1.99 \%$, respectively, which were significantly higher than that of the negative control (5.03 $\pm 0.22 \%)$. Similarly, the RNAi inhibition rates of SEC-4 (72.98 $\pm 2.97 \%)$ and SEC-7 $(82.90 \pm 3.15 \%)$ in HeLa cells were significantly different from that of the negative control (3.95 \pm $0.33 \%$ ). However, the results demonstrated that the inhibition rate of RNAi ranged from $42 \%$ to $81 \%$, indicating that not all target sites were very effective. For the sites inside the open reading frame, SEC-4 and SEC-5 showed the highest efficiency. It is possible that the two target sites fell on hTERT V867M and R901W variants, which have been demonstrated to be associated with telomere shortening (20, 21). For the 3'-UTR sites, SEC-7 and SEC-8 showed the highest efficiency, indicating that 3'-UTR plays an important role in RNAi through not only miRNA, but also siRNA.

In summary, we successfully constructed hTERT-specific SECs by overlap extension PCR and demonstrated that hTERT-specific SECs significantly inhibited telomerase activity in HepG2 cells. Our strategy of producing SECs would provide a novel and promising method to screen effective RNAi-targeted sequences and would accelerate development of nonviral cancer gene therapies.

\section{Authors' Contributions}

Jian Xiong Peng developed the original idea and the protocol, supervised the whole study. Hui Xu abstracted and analyzed data, wrote the manuscript and revised the manuscript for important intellectual content. Xia Gong and Hui Hui Zhang contributed to the development of the protocol and prepared the manuscript. Qin Zhang and Dandan Zhao provided administrative, technical and material support.

\section{Funding/Support}

This work was supported by a grant from the National Natural Science Foundation of China (98SSY1003).

\section{References}

1. Kim NW, Piatyszek MA, Prowse KR, Harley CB, West MD, Ho PL, et al. Specific association of human telomerase activity with immortal cells and cancer. Science.1994;266(5193):2011-5.

2. Noureini SK, Wink M. Antiproliferative effect of the isoquinoline alkaloid papaverine in hepatocarcinoma HepG-2 cells-inhibition of telomerase and induction of senescence. Molecules. 2014;19(8):11846-59.

3. Zhao L, Wink M. The beta-carboline alkaloid harmine inhibits telomerase activity of MCF-7 cells by down-regulating hTERT mRNA expression accompanied by an accelerated senescent phenotype. PeerJ. 2013;1.

4. Noureini SK, Wink M. Antiproliferative effects of crocin in HepG2 cells by telomerase inhibition and hTERT down-regulation. Asian Pac J Cancer Prev. 2012;13(5):2305-9.

5. Rao Y, Xiong W, Liu H, Jia C, Zhang H, Cui Z, et al. Inhibition of telomerase activity by dominant-negative hTERT retards the growth of breast cancer cells. [[Epub ahead of print]];Breast Cancer. 2014

6. Imani Fooladi AA, Aghelimansour A, Nourani MR. Evaluation of the Pathogenesis of Pseudomonas aeruginosa's Flagellum Before and After Flagellar Gene Knockdown by Small Interfering RNAs (siRNA). Jundishapur J Microbiol. 2013;6(3):273-8.

7. Amarzguioui M, Rossi JJ, Kim D. Approaches for chemically synthesized siRNA and vector-mediated RNAi. FEBS Lett. 2005;579(26):5974-81.

8. Castanotto D, Li H, Rossi JJ. Functional siRNA expression from transfected PCR products. RNA. 2002;8(11):1454-60.

9. Gou D, Jin N, Liu L. Gene silencing in mammalian cells by PCRbased short hairpin RNA. FEBS Lett. 2003;548(1-3):113-8.

10. Tran N, Cairns MJ, Dawes IW, Arndt GM. Expressing functional siRNAs in mammalian cells using convergent transcription. BMC Biotechnol. 2003;3:21.

11. Sambrook J, Russell DW. Molecular Cloning: A Laboratory Manual. 3rd ed: Cold Spring Harbor Laboratory Pres; 2001.

12. Kim NW, Wu F. Advances in quantification and characterization of telomerase activity by the telomeric repeat amplification protocol (TRAP). Nucleic Acids Res. 1997;25(13):2595-7.

13. Uehara H. Real-time detection and quantification of telomerase activity utilizing energy transfer primers. Methods Mol Biol. 2006;335:157-69.

14. El-Saeed GK, El-Bassuoni MA, El-Saeed HH, Gaweesh IA, Soliman MA, Attiya H. Molecular Detection of Human Telomerase mRNA (hTERT-mRNA) in Egyptian Patients with Hepatocellular Carcinoma. Hepat Mon. 2009;9(2):114-21.

15. Zhang W, Xing L. RNAi gene therapy of SiHa cells via targeting human TERT induces growth inhibition and enhances radiosensitivity. Int J Oncol. 2013;43(4):1228-34.

16. Cheng W, Wei Z, Gao J, Zhang Z, Ge J, Jing K, et al. Effects of combined siRNA-TR and -TERT on telomerase activity and growth of bladder transitional cell cancer BIU-87 cells. J Huazhong Univ Sci Technolog Med Sci. 2010;30(3):391-6.

17. Fang L, Cheng Q, Li W, Liu J, Li L, Xu K, et al. Antitumor activities of an oncolytic adenovirus equipped with a double siRNA targeting Ki67 and hTERT in renal cancer cells. Virus Res. 2014;181:61-71.

18. Chen M, Zhang L, Zhang HY, Xiong X, Wang B, Du Q, et al. A universal plasmid library encoding all permutations of small interfering RNA. Proc Natl Acad Sci U S A. 2005;102(7):2356-61.

19. Jian R, Peng T, Deng S, Jiang J, Hu F, An J, et al. A simple strategy for generation of gene knockdown constructs with convergent $\mathrm{H} 1$ and U6 promoters. Eur J Cell Biol. 2006;85(5):433-40.

20. Alder JK, Cogan JD, Brown AF, Anderson CJ, Lawson WE, Lansdorp $\mathrm{PM}$, et al. Ancestral mutation in telomerase causes defects in repeat addition processivity and manifests as familial pulmonary fibrosis. PLoS Genet. 2011;7(3).

21. Marrone A, Walne A, Tamary H, Masunari Y, Kirwan M, Beswick R, et al. Telomerase reverse-transcriptase homozygous mutations in autosomal recessive dyskeratosis congenita and HoyeraalHreidarsson syndrome. Blood. 2007;110(13):4198-205. 